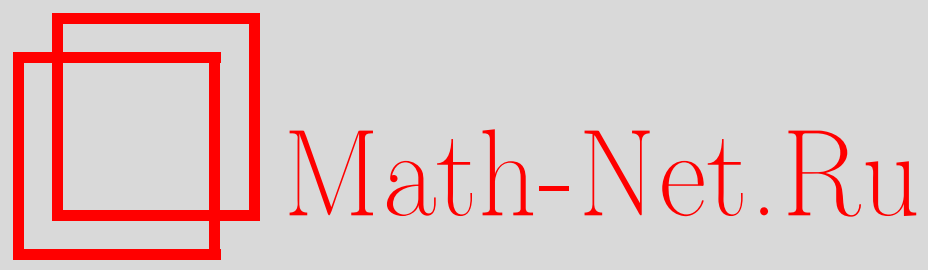

О. М. Мясниченко, Прямое произведение раскрытых ласточкиных хвостов в задаче об обходе препятствий, Функи. анализ и его прил., 1996, том 30, выпуск 4, 10-18

DOI: https://doi.org/10.4213/faa546

Использование Общероссийского математического портала MathNet.Ru подразумевает, что вы прочитали и согласны с пользовательским соглашением

http://www . mathnet.ru/rus/agreement

Параметры загрузки:

IP : 52.87 .193 .239

26 апреля 2023 г., 15:33:39

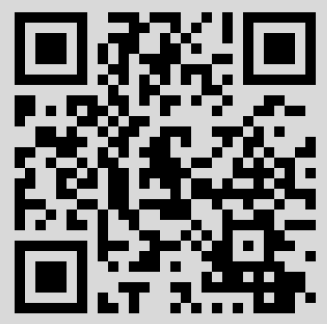


Функииональньй анализ и его приложения

1996, т. 30, вып. 4, с. 10-18

УДК 514.853

\title{
Прямое произведение раскрытых ласточкиных хвостов в задаче об обходе препятствий ${ }^{\star}$
}

\author{
(c) 1996. О. М. Мясниченко
}

В работах [1-4] и других исследовалась задача об обходе препятствия - задача исследования лагранжевых многообразий, которые состоят из экстремалей функционала действия гамильтоновой системы, обходящих препятствие, ограниченное гладкой гиперповерхностью в конфигурационном многообразии.

Рассмотрим, например, евклидово пространство $M$ и препятствие в нем, край которого - гладкая гиперповерхность Г. Огибающий препятствие кратчайший путь между двумя точками $u, v \in M$ в простейшем случае состоит из отрезка луча (ориентированной прямой) $l_{1}$, проходящего через начальную точку $u$ и касающегося $\Gamma$, отрезка геодезической $l_{2} \subset \Gamma$, стартующей в направлении $l_{1}$ из точки касания луча $l_{1}$ и гиперповерхности $\Gamma$, и отрезка луча, касающегося $l_{2}$ и проходящего через конечную точку $v$. Рассматривая пути, соединяющие точку $u$ и точки из некоторой окрестности точки $v$, получим семейство проходящих через точку $u$ и касающихся гиперповерхности $\Gamma$ лучей, семейство стартующих из точек касания геодезических на Г и семейство срывающихся с них лучей. Семейство геодезических на $Г$ задает лагранжево подмногообразие $L_{\Gamma} \subset T^{*} \Gamma$ : принадлежащая $L_{\Gamma}$ точка - это точка на геодезической и касательный к этой геодезической в этой точке единичный вектор (векторы и ковекторы отождествляем с помощью метрики). Многообразие $L_{\Gamma}$ называем многообразием, состоящим из стартующих из точек касания геодезических. Образ $l_{\Gamma}$ многообразия $L_{\Gamma}$ в пространстве всех ориентированных геодезических на $Г$ называем многообразием геодезических, стартующих из точек касания. Аналогично, семейство лучей, касающихся геодезических, задает лагранжево многообразие $L_{M} \subset T^{*} M$, называемое многообразием, состоящим из лучей, срывающихся с пучка геодезических, задаваемого многообразием $l_{\Gamma}$. Образ $l_{M}$ многообразия $L_{M}$ в пространстве всех ориентированных прямых в $M$ называем многообразием лучей, срывающихся с пучка геодезических, задаваемого многообразием $l_{\Gamma}$.

Лагранжевы многообразия, появляющиеся в задаче об обходе препятствия, в общем случае являются особыми [1-4], причем типичная особенность - раскрытый ласточкин хвост.

Раскрытым ласточкиным хвостом $\tau_{n}$ (размерности $n$ ) называется особое лагранжево подмногообразие симплектического пространства многочленов вида

$$
\frac{x^{2 n+1}}{(2 n+1) !}+Q_{1} \frac{x^{2 n-1}}{(2 n-1) !}+\cdots+Q_{n} \frac{x^{n}}{n !}-P_{n} \frac{x^{n-1}}{(n-1) !}+\cdots+(-1)^{n} P_{1}
$$

* Работа выполнена при финансовой поддержке Российского фонда фундаментальных исследований, грант 94-01-00255, и фонда NWO, грант 47.03.005. 
$(Q, P$ - координаты Дарбу), образованное многочленами, имеющими корень кратности $>n$. Многообразие $\tau_{n, k}=\tau_{n-k} \times \mathbb{R}^{k}$ называется $k$-мерной надстройкой над $\tau_{n-k}[2]$.

В [2] доказано, что если $l_{\Gamma}$ гладко, то многообразие $l_{M}$ общего положения в некоторой окрестности луча, имеющего $k$-й порядок касания с краем препятствия, симплектоморфно $\tau_{n+k-1, n}(k+n=\operatorname{dim} M)$.

Предположение о гладкости многообразия $l_{\Gamma}$ справедливо не всегда [4]: многообразие $l_{\Gamma}$ общего положения в некоторой окрестности геодезической, стартующей из точки касания порядка $k$ луча с краем, симплектоморфно $\tau_{m+k-1, m}$ $(k+m=\operatorname{dim} \Gamma)$.

Основной результат данной работы состоит в следующем:

(1) Многообразие лучей общего положения, срывающихся с пучка геодезических, задаваемого многообразием $l_{\Gamma}$, в некоторой окрестности имеюшего 2-й порядок касания с краем препятствия луча формально симплектоморфно $\tau_{1} \times l_{\Gamma}$.

(2) Многообразие лучей общего положения, срываюшихся с пучка геодезических, задаваемого многообразием $l_{\Gamma}$, которое локально диффеоморфно $l \times \mathbb{R}$, где $l$ - некоторое аналитическое множество, в некоторой окрестности луча, имеющего 3-й порядок касания с краем препятствия, формально диффеоморфно $\tau_{2} \times l$.

Многообразие $l_{\Gamma}$ общего положения диффеоморфно прямому произведению $l \times \mathbb{R}$ в некоторой окрестности геодезической, с которой срывается луч, имеющий 3-й порядок касания с краем препятствия. Исключением является случай, когда $\operatorname{dim} M=4$ и рассматриваемая точка срыва является точкой касания падающего на край препятствия луча, имеющего 3-й порядок касания с краем (т.е. случай, когда срывающийся с края луч есть продолжение луча, падающего на край).

Из результатов $[1,4]$ и данной работы вытекает локальная симплектическая классификация особенностей двумерных многообразий лучей, встречающихся в типичных задачах об обходе препятствия в трехмерном пространстве. Помимо самопересечений, это $\tau_{2}[1], \tau_{1} \times \tau_{1}, \tau_{1} \times \mathbb{R}[1]$ и $\mathbb{R}^{2}$. В типичных четырехмерных задачах встречаются особенности $\tau_{3}[2], \tau_{2} \times \tau_{1}, \tau_{2} \times \mathbb{R}[1], \tau_{1} \times \tau_{2}, \tau_{1} \times \tau_{1} \times \mathbb{R}$, $\tau_{1} \times \mathbb{R}^{2}[1], \mathbb{R}^{3}$ и (в изолированных точках) первая нерасклассифицированная особенность в некоторой окрестности луча, имеющего третий порядок касания с краем и являющегося продолжением падающего на край луча. Кроме того, $\tau_{2} \times \tau_{1}$ - гладкая (не симплектическая) нормальная форма.

\section{§1. Определения и формулировки}

Пусть $M$ - гладкое конфигурационное многообразие с краем $\Gamma=$ $\{q \in M \mid f(q)=0\}$ гамильтоновой системы с гамильтонианом $h, T^{*} M-$ пространство кокасательного расслоения, $\omega$ - стандартная симплектическая структура на $T^{*} M, \pi: T^{*} M \rightarrow M$ - проекция кокасательного расслоения и $F=\pi^{*} f$. В $T^{*} M$ рассмотрим две гиперповерхности

$$
H=\left\{x \in T^{*} M \mid h(x)=0\right\} \quad \text { и } \quad T_{\Gamma}^{*} M=\left\{x \in T^{*} M \mid F(x)=0\right\} .
$$

Обозначим естественную проекцию вдоль характеристик гиперповерхности $T_{\Gamma}^{*} M$ через $\rho: T_{\Gamma}^{*} M \rightarrow T^{*} \Gamma$ (многообразие характеристик гиперповерхности $T_{\Gamma}^{*} M$ 
естественно симплектоморфно многообразию $T^{*} \Gamma$ [1]: характеристика гиперповерхности $T_{\Gamma}^{*} M$ образована ковекторами в точке из $\Gamma$, ограничения которых на касательное пространство к Г в этой точке совпадают; эти ограничения задают ковектор в точке из $\Gamma$, в который отображается рассматриваемая характеристика). Через $\chi: H \rightarrow N$ обозначим естественную проекцию вдоль характеристик гиперповерхности $H$ (локально $N$ является гладким симплектическим многообразием).

Лагранжево подмногообразие, принадлежащее некоторой гиперповерхности фазового пространства, состоит из характеристик данной гиперповерхности (интегральных кривых поля косоортогональных дополнений касательных пространств гиперповерхности), т.е. из экстремалей соответствующего функционала действия. Лагранжевы многообразия, состоящие из экстремалей функщионала действия гамильтоновой системы с односторонним ограничением, описываются следующим образом:

Пусть $B \subset M$ - некоторое гладкое подмногообразие (начальный фронт). Рассмотрим $L_{B} \subset T^{*} M$ - гладкое лагранжево подмногообразие, состоящее из ковекторов в точках из $B$, нулевых на касательных к $B$ плоскостях. Обозначим через $L$ объединение характеристик гиперповерхности $H$, проходящих через точки пересечения $L_{B} \cap H$. Для общего начального фронта многообразие $L_{B}$ трансверсально $H$; следовательно, $L$ - гладкое (во всяком случае, локально) лагранжево подмногообразие пространства $T^{*} M$. Если $B$ и $\Gamma-$ подмногообразия общего положения, то $L$ пересекает $T_{\Gamma}^{*} M$ трансверсально, а значит, $l=T_{\Gamma}^{*} M \cap L$ гладкое и не касается характеристик гиперповерхности $T_{\Gamma}^{*} M$; поэтому $\rho(l)$ есть гладкое лагранжево подмногообразие многообразия $T^{*} \Gamma$. Рассмотрим множества $H^{\prime}$ и $H^{\prime \prime}$ критических точек и критических значений ограничения проекции $\rho$ на $H \cap T_{\Gamma}^{*} M$. Обозначим через $\chi^{\prime \prime}: H^{\prime \prime} \rightarrow N^{\prime \prime}$ проекцию вдоль характеристик гиперповерхности $H^{\prime \prime}$, через $L_{\Gamma}$ - объединение пересекающих $\rho(l) \cap H^{\prime \prime}$ характеристик гиперповерхности $H^{\prime \prime}$, через $l_{\Gamma}-$ образ $L_{\Gamma}$ в $N^{\prime \prime}$ и через $L_{M}$ - объединение характеристик гиперповерхности $H$, проходящих через точки пересечения $H \cap \rho^{-1}\left(L_{\Gamma}\right)$.

Рассмотрим $T_{\Gamma}^{*} M \cap H=H^{(0)} \supset H^{(1)} \supset H^{(2)} \supset \cdots-$ фильтрацию по степени касания характеристик гиперповерхности $H$ с гиперповерхностью $T_{\Gamma}^{*} M$; здесь $H^{(i)}$ образовано точками, в которых характеристическое направление гиперповерхности $H$ (задаваемое гамильтоновым векторным полем с гамильтонианом $h$ ) касается подмногообразия $H^{(i-1)}$, т.е.

$$
H^{(i)}=\left\{x \in T^{*} M \mid F(x)=h(x)=\langle F, h\rangle(x)=\cdots=\langle\cdots\langle F, h\rangle, \ldots, h\rangle(x)=0\right\}
$$

$(i$ раз скобка Пуассона $\langle\cdot, \cdot\rangle)$. Нетрудно видеть, что

$$
H^{\prime}=H^{(1)}=\left\{x \in T^{*} M \mid F(x)=h(x)=\langle F, h\rangle(x)=0\right\} .
$$

Заметим, что ограничение проекции $\chi$ (имеющей одномерные слои) на $H^{(0)}$ (гиперповерхность в $H$ ) в случае общего положения имеет только особенности типа $A_{k}$ : в некоторой окрестности любой точки из $H^{(k)} \backslash H^{(k+1)}$ ограничение $\chi$ на $H^{(0)}$ в некоторых координатах $\left(x, \lambda_{1}, \ldots, \lambda_{n}\right)$ на $H$ имеет вид

$$
\left\{\left(x, \lambda_{1}, \ldots, \lambda_{n}\right) \mid x^{k+1}+x^{k-1} \lambda_{1}+\cdots+\lambda_{k}=0\right\} \mapsto\left(\lambda_{1}, \ldots, \lambda_{n}\right) .
$$


ПРимеР. В рассматривавшемся выше примере о минимизации длины пути в евклидовом пространстве гиперповерхность $H$ есть множество всех единичных ковекторов, $H=\left\{(q, p) \in T^{*} M \mid\|p\|=1\right\}, H^{(0)}$ - множество единичных (ко)векторов в точках из $\Gamma, H^{(1)}$ — множество единичных (ко)векторов, касающихся $\Gamma, H^{(2)} \backslash H^{(3)}$ - множество единичных (ко)векторов, имеющих второй порядок касания с $\Gamma, H^{(3)} \backslash H^{(4)}$ - множество единичных (ко)векторов, имеющих третий порядок касания с $\Gamma$, и т.д. Начальный фронт $B$ - это начальная точка $u$, многообразие $L$ состоит из лучей, проходящих через начальную точку, $L=\left\{(q, p) \in T^{*} M \mid q\right.$ - точка луча, проходящего через точку $u$, $p$ - единичный (ко)касательный вектор к этому лучу в точке $q\}$. Многообразие $l$ соответствует лучам, проходящим через точку $u$ и пересекающим $\Gamma$, $l=\{(q, p) \in L \mid q \in \Gamma\}$, а пересечение $l \cap H^{\prime}-$ касающимся $\Gamma$ лучам, $l \cap H^{\prime}=\{(q, p) \in l \mid$ луч $q+p t, t \in \mathbb{R}$, касается $\Gamma\}$. Многообразие $L_{\Gamma}$ - это объединение геодезических на $\Gamma$, стартующих из точек касания, а $L_{M}$ - объединение лучей, срывающихся с соответствующего пучка геодезических на $\Gamma$.

Используя введенную выше терминологию, назовем проекции в $M$ характеристик гиперповерхности $H$ (для любого гамильтониана $h$ ) лучами, а проекции в Г характеристик гиперповерхности $H^{\prime \prime}$ - геодезическими.

ОПРЕДЕЛЕНИЕ. Многообразие $l_{\Gamma}$ мы называем многообразием геодезических, стартуюших из точек касания.

ОПРЕДЕЛЕНИЕ. Многообразие $l_{M}$ мы называем многообразием лучей, срываюшихся с пучка геодезических, задаваемого многообразием $l_{\Gamma}$.

Основным результатом, на который опирается данная работа, является

Теорема 1 (Арнольд [1]). В некоторой окрестности точки z, в которой ограничение $\chi$ на $H^{(0)}$ имеет особенность $A_{k}$, пара $\left(H, H^{(0)}\right)$ обшего положения формальным (как минимум) симплектоморфизмом приводится $к$

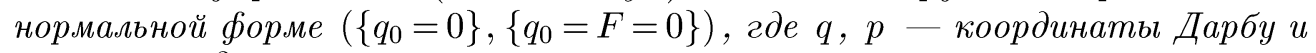

(a) $F=p_{0}^{2}+p_{1}$ npu $k=1$;

(b) $F=p_{0}^{3}+p_{1} p_{0}+q_{1}$ npu $k=2$;

(c) $F=p_{0}^{4}+p_{1} p_{0}^{2}+q_{2} p_{0}+p_{2}$ npu $k=3$.

ЗАмЕчАния. 1. Случай (а) получен в [5]. Пара $\left(H, H^{(0)}\right)$ приводится к нормальной форме гладким симплектоморфизмом.

2. Условия общности положения теоремы 1 требуют:

- в случае (b) невырожденности ограничения симплектической структуры пространства характеристик гиперповерхности $H$ на касательное пространство в точке $\chi(z)$ к ребру возврата множества критических значений ограничения $\chi$ на $H^{(0)}$ (это множество локально диффеоморфно цилиндру подходящей размерности над полукубической параболой);

- в случае (c) (когда множество критических значений ограничения $\chi$ на $H^{(0)}$ локально диффеоморфно цилиндру над ласточкиным хвостом - многообразием многочленов $x^{4}+\lambda_{1} x^{2}+\lambda_{2} x+\lambda_{3}$ от $x$, имеющих кратный корень) невырожденности ограничения симплектической структуры пространства характеристик гиперповерхности $H$ на касательное пространство в точке $\chi(z)$ к подмногообразию $\lambda_{1}=\lambda_{2}=0$. 
Предположим, что гамильтониан $h$ является квадратичным и выпуклым по импульсам.

Теорема 2. В случае общего положения многообразие лучей, срываюшихся с пучка геодезических, задаваемого лагранжевым многообразием $l_{\Gamma}$, в некоторой окрестности имеющего 2-й порядок касания с краем луча формально симплектоморфно $\tau_{1} \times l_{\Gamma}$.

Рассмотрим случай, когда срываюшийся луч имеет третий порядок касания с краем, $x \in H^{(3)} \backslash H^{(4)}$ — точка срыва, $\rho(x) \in L_{\Gamma}$. Предположим, что многообразие $L_{\Gamma}$ в точке $\rho(x)$ локально диффеоморфно $l \times \mathbb{R}^{m}$ (пишем $L_{\Gamma} \cong l \times \mathbb{R}^{m}$ ), $m>1$, где $l$ - некоторое аналитическое множество. В этом случае многообразие $l_{\Gamma}$ в точке $\chi^{\prime \prime}(\rho(x))$ локально диффеоморфно произведению $l \times \mathbb{R}^{m-1}$, $m>1$.

ТЕОрема 3. В случае общего положения многообразие лучей, срываюшихся с пучка геодезических, задаваемого лагранжевым многообразием $l_{\Gamma} \cong$ $l \times \mathbb{R}^{m-1}, m>1$, в некоторой окрестности имеющего 3 -й порядок касания с краем луча формально диффеоморфно $\tau_{2} \times l \times \mathbb{R}^{m-2}$.

ЗАмЕчАния. 1. Условия общности положения теоремы 2 заключаются в следующем:

a) ограничение проекции $\chi$ на $H^{(0)}$ в некоторой окрестности точки срыва $x$ (точки, через которую проходит характеристика гиперповерхности $H$, проектирующаяся в рассматриваемый луч) имеет особенность $A_{2}$;

b) пара $\left(H, H^{(0)}\right)$ удовлетворяет условиям общности положения теоремы 1 , п. (b) (сформулированным в замечании, следующем за этой теоремой).

2. Условия общности положения теоремы 3 заключаются в следующем:

a) ограничение $\chi$ на $H^{(0)}$ в некоторой окрестности рассматриваемой точки срыва $x \in H^{(3)} \backslash H^{(4)}, \rho(x) \in L_{\Gamma}$, имеет особенность $A_{3}$;

b) пара $\left(H, H^{(0)}\right)$ удовлетворяет условиям общности положения теоремы 1 , п. (c);

c) в точке $\rho(x)=\left(y_{1}, y_{2}\right) \in l \times \mathbb{R}^{m}\left(\cong L_{\Gamma}\right)$ ребро $y_{1} \times \mathbb{R}^{m}$ трансверсально в $H^{\prime \prime}$ подмногообразию $\rho\left(H^{(3)}\right) \subset H^{\prime \prime}$.

3. Если срывающийся с края луч не является продолжением падающего на край луча, имеющего с краем максимальный порядок касания (равный размерности края), то в случае общего положения $L_{\Gamma} \cong l \times \mathbb{R}^{m}, m>1$. Действительно, многообразие $L_{\Gamma}$ локально диффеоморфно $\tau_{n, k}, k>0$, причем $k=1$ только вдоль отдельных геодезических [4]. В общей точке такой «самой вырожденной» изолированной геодезической касающийся ее вектор имеет первый порядок касания с краем, в изолированных точках — второй порядок и в случае общего положения нигде, за исключением точки касания, не имеет касания порядка, большего двух. То есть если срывающаяся геодезическая имеет третий порядок касания с краем, то соответствующий пучок геодезических задается многообразием $L_{\Gamma} \cong \tau_{n-2, k-2} \times \mathbb{R}^{2}, k>1$.

Из результатов [4] о нормальных формах многообразий $L_{\Gamma}$ следует, что на уровне формальных рядов справедливо

СлЕдСТВИЕ 4. В случае общего положения многообразие геодезических на крае локально симплектоморфно $\tau_{n-1, k-1}(n=\operatorname{dim} \Gamma)$ для некоторого 
$k>0$, м многообразие лучей, срыввююихся с соответствующего пучка геодезических,

(а) в некоторой окрестности луча, имеющего второй порядок касания $c$ краем, симплектоморфно прямому произведению $\tau_{1} \times \tau_{n-1, k-1}$;

(b) в некоторой окрестности луча, имеющего третий порядок касания с краем, диффеоморфно прямому произведению $\tau_{2} \times \tau_{n-2, k-2}$, если $k>1$.

\section{§2. Доказательства}

Предварительные результаты. Гамильтониан $h$ квадратичен и выпукл по импульсам; следовательно, $\rho^{-1}\left(H^{\prime \prime}\right) \cap H^{(0)}=H^{\prime}\left(=H^{(1)}\right)$ и в точках из $H^{\prime}$ ограничение проекции $\rho$ на $H \cap T_{\Gamma}^{*} M$ имеет особенность единственного типа $A_{1}$, откуда следует, что $H^{\prime \prime}$ - гладкая гиперповерхность в $T^{*} \Gamma$ и ограничение $\rho$ на $H^{\prime}$ — диффеоморфизм. Рассмотрим ограничение симплектической формы $\omega$ на $H^{(1)}$ и поле ядер этого ограничения в касательных к $H^{(1)}$ пространствах. Нетрудно видеть, что это поле - невырожденное поле направлений, которые мы будем называть характеристическими. Действительно, одномерность ядер ограничения формы $\omega$ на $H^{(1)}$ следует из того, что производная диффеоморфизма $\left.\rho\right|_{H^{(1)}}$ (ограничения $\rho$ на $\left.H^{(1)}\right)$ отображает это поле ядер в невырожденное поле характеристических направлений гладкой гиперповерхности $H^{\prime \prime} \subset T^{*} \Gamma$ (что, в свою очередь, следует из естественной симплектоморфности многообразия характеристик гиперповерхности $T_{\Gamma}^{*} M \subset T^{*} M$ и многообразия $T^{*} \Gamma$, а именно $\rho^{*} \omega_{\Gamma}=\left.\omega\right|_{T_{\Gamma}^{*} M}$, где $\omega_{\Gamma}$ - естественная симплектическая структура на $T^{*} \Gamma$ ). Интегральные кривые поля характеристических направлений назовем характеристиками подмногообразия $H^{(1)}$. Пространство характеристик подмногообразия $H^{(1)}$ наделяется естественной симплектической структурой, задаваемой следующим условием: прообраз симплектической формы (относительно проекции вдоль характеристик подмногообразия $H^{(1)}$ ) равен ограничению формы $\omega$ на $H^{(1)}$.

ПРЕДЛОЖЕНИЕ 5. Пространства характеристик гиперповерхности $H^{\prime \prime}$ и подмногообразия $H^{(1)}$ естественно симплектоморфны.

ДокАЗАтЕльство. Диффеоморфизм $\left.\rho\right|_{H^{(1)}}$ отправляет характеристики подмногообразия $H^{(1)}$ в характеристики гиперповерхности $H^{\prime \prime}$, индуцируя диффеоморфизм пространств характеристик. Из согласованности $\left.\rho\right|_{H^{(1)}}$ с ограничением $\omega$ на $H^{(1)}$ и $\omega_{\Gamma}$ на $H^{\prime \prime}$ следует, что этот диффеоморфизм пространств характеристик является симплектоморфизмом.

ДОкАЗАТЕЛЬСТВо ТЕОРЕмЫ 2 . Обозначим через $l_{H}$ пересечение $H$ с множеством проектирующихся в $L_{\Gamma}$ характеристик гиперповерхности $T_{\Gamma}^{*} M, l_{H}=$ $\rho^{-1}\left(L_{\Gamma}\right) \cap H$. Многообразие $l_{H}$ изотропно. Из невырожденности ограничения $\rho$ на $H^{\prime}\left(=H^{(1)}\right)$ и из того, что $L_{\Gamma} \subset H^{\prime \prime}$, следует, что $l_{H}=\left(\left.\rho\right|_{H^{(1)}}\right)^{-1}\left(L_{\Gamma}\right)$. Таким образом, $l_{H}$ - изотропное подмногообразие в $T^{*} M$, диффеоморфное $L_{\Gamma}$ и состоящее из характеристик подмногообразия $H^{(1)}$, проекция которого в пространство характеристик этого подмногообразия симплектоморфна лагранжеву подмногообразию $l_{\Gamma} \subset T^{*} \Gamma$. Из условия асимптотичности касания срывающегося с Г луча и из п. (b) теоремы 1 следует, что в некоторой окрестности точки 
$x \in l_{H}$, через которую проходит рассматриваемая характеристика гиперповерхности $H$, пара $\left(H, H^{(0)}\right)$ формальным симплектоморфизмом приводится к виду $\left(\left\{q_{0}=0\right\},\left\{q_{0}=p_{0}^{3}+p_{1} p_{0}+q_{1}=0\right\}\right)$. Имеем

$$
\begin{aligned}
H\left(=\left\{q_{0}=0\right\}\right) & \supset H^{(0)}\left(=\left\{q_{0}=p_{0}^{3}+p_{1} p_{0}+q_{1}=0\right\}\right) \\
& \supset H^{(1)}\left(=\left\{q_{0}=0, p_{1}=-3 p_{0}^{2}, q_{1}=2 p_{0}^{3}\right\}\right) \\
& \supset H^{(2)}\left(=\left\{q_{0}=q_{1}=p_{0}=p_{1}=0\right\}\right),
\end{aligned}
$$

т. е. функции $\left(p_{0}, q_{2}, \ldots, q_{n}, p_{2}, \ldots, p_{n}\right)=\left(p_{0}, q^{\prime}, p^{\prime}\right)$ образуют координатную систему на $H^{(1)},\left.\omega\right|_{H^{(1)}}=d p_{2} \wedge d q_{2}+\cdots+d p_{n} \wedge d q_{n}$, поле ядер ограничения $\left.\omega\right|_{H^{(1)}}$ задается векторным полем $\partial / \partial p_{0}$ и функции $\left(q^{\prime}, p^{\prime}\right)$ могут рассматриваться в качестве координат Дарбу на пространстве характеристик подмногообразия $H^{(1)}$. В этих координатах $l_{H}=\left\{\left(p_{0}, q^{\prime}, p^{\prime}\right) \mid\left(q^{\prime}, p^{\prime}\right) \in l_{\Gamma}\right\}$ (здесь $l_{\Gamma}$ обозначает образ $l_{H}$ в пространстве характеристик подмногообразия $H^{(1)}$; согласно предложению 5 , этот образ симплектоморфен «настоящему» $\left.l_{\Gamma} \subset N^{\prime \prime}\right)$. Проектируя $l_{H}$ вдоль характеристик гиперповерхности $H$ (забывая $p_{0}$ ), получим

$$
l_{M}=\chi\left(l_{H}\right)=\left\{\left(q_{1}, p_{1}, q^{\prime}, p^{\prime}\right) \mid 4 p_{1}^{3}+27 q_{1}^{2}=0,\left(q^{\prime}, p^{\prime}\right) \in l_{\Gamma}\right\},
$$

что и доказывает теорему.

ДокаЗАТЕЛЬСтво теОРемы 3 . Рассмотрим точку $x$, через которую проходит характеристика гиперповерхности $H$, проектирующаяся в луч, имеющий 3-й порядок касания с краем, $x \in l_{H}=\rho^{-1}\left(L_{\Gamma}\right) \cap H, x \in H^{(3)} \backslash H^{(4)}$. Из п. (c) теоремы 1 следует, что в некоторой окрестности точки $x$ пара $\left(H, H^{(0)}\right)$ формально симплектоморфна паре $\left(\left\{q_{0}=0\right\},\left\{q_{0}=p_{0}^{4}+p_{1} p_{0}^{2}+p_{0} q_{2}+p_{2}=0\right\}\right)$. Имеем

$$
\begin{aligned}
H\left(=\left\{q_{0}=0\right\}\right) & \supset H^{(0)}\left(=\left\{q_{0}=p_{0}^{4}+p_{1} p_{0}^{2}+p_{0} q_{2}+p_{2}=0\right\}\right) \\
& \supset H^{(1)}\left(=\left\{q_{0}=0, q_{2}=-4 p_{0}^{3}-2 p_{1} p_{0}, p_{2}=3 p_{0}^{4}+p_{1} p_{0}^{2}\right\}\right) \\
& \supset H^{(2)}\left(=\left\{q_{0}=0, p_{1}=-6 p_{0}^{2}, q_{2}=8 p_{0}^{3}, p_{2}=-3 p_{0}^{4}\right\}\right) \\
& \supset H^{(3)}\left(=\left\{q_{0}=p_{0}=p_{1}=p_{2}=q_{2}=0\right\}\right) .
\end{aligned}
$$

Функции $\left(p_{0}, q_{1}, p_{1}, q_{3}, \ldots, q_{n}, p_{3}, \ldots, p_{n}\right)=\left(p_{0}, q_{1}, p_{1}, q^{\prime \prime}, p^{\prime \prime}\right)$ образуют координатную систему на $H^{(1)}$, ограничение симплектической структуры $\omega$ на $H^{(1)}$ равно $\omega^{\prime}=\left.\omega\right|_{H^{(1)}}=d p_{1} \wedge d\left(q_{1}+12 p_{0}^{5} / 5+2 p_{1} p_{0}^{3} / 3\right)+d p^{\prime \prime} \wedge d q^{\prime \prime}$, или $\omega^{\prime}=d p_{1} \wedge d Q_{1}+d p^{\prime \prime} \wedge d q^{\prime \prime}$ после замены $Q_{1}=q_{1}+12 p_{0}^{5} / 5+2 p_{1} p_{0}^{3} / 3$. Поле ядер ограничения $\left.\omega\right|_{H^{(1)}}$ задается векторным полем $\partial / \partial p_{0}$, функции $\left(Q_{1}, q^{\prime \prime}, p_{1}, p^{\prime \prime}\right)$ могут рассматриваться в качестве координат Дарбу на пространстве характеристик подмногообразия $H^{(1)}$ (и, согласно предложению 5 , на пространстве характеристик гиперповерхности $\left.H^{\prime \prime}\right)$. В этих координатах многообразие $l_{H}$ имеет вид $l_{H}=\left\{\left(p_{0}, Q_{1}, q^{\prime \prime}, p_{1}, p^{\prime \prime}\right) \mid\left(Q_{1}, q^{\prime \prime}, p_{1}, p^{\prime \prime}\right) \in l_{\Gamma}\right\}$. Из условия $L_{\Gamma} \cong l \times \mathbb{R}^{m}$, $m>1$, следует, что $l_{\Gamma} \cong l \times \mathbb{R}^{m-1}$. Многообразие $l$ аналитическое, т. е. $l_{\Gamma}$ задается некоторой системой аналитических уравнений $\psi_{1}\left(Q_{1}, p_{1}, q^{\prime \prime}, p^{\prime \prime}\right)=\cdots=$ $\psi_{r}\left(Q_{1}, p_{1}, q^{\prime \prime}, p^{\prime \prime}\right)=0$, где хотя бы одна из функций $\psi_{i}$ невырожденна.

Из условий, что $L_{\Gamma} \cong l \times \mathbb{R}^{m}, m>1$, и что в точке $\rho(x)=\left(y_{1}, y_{2}\right) \in l \times \mathbb{R}^{m}$ ребро $y_{1} \times \mathbb{R}^{m}$ трансверсально $\rho\left(H^{(3)}\right)$, следует, что $l_{H} \cong l \times \mathbb{R}^{m}, m>1$, и в 
точке $x=\left(x_{1}, x_{2}\right) \in l \times \mathbb{R}^{m}$ ребро $x_{1} \times \mathbb{R}^{m}$ трансверсально подмногообразию $H^{(3)} \subset H^{(1)}, H^{(3)}=\left\{p_{0}=p_{1}=0\right\}$.

ПРЕДЛОЖЕНИЕ 6.

$$
\begin{aligned}
T_{x}\left(x_{1} \times \mathbb{R}^{m}\right) \subset \operatorname{span}\left\{\frac{\partial}{\partial p_{0}}, \frac{\partial \psi_{i}}{\partial p_{1}}\right. & (x) \frac{\partial}{\partial Q_{1}}+\frac{\partial \psi_{i}}{\partial p^{\prime \prime}}(x) \frac{\partial}{\partial q^{\prime \prime}} \\
& \left.-\frac{\partial \psi_{i}}{\partial Q_{1}}(x) \frac{\partial}{\partial p_{1}}-\frac{\partial \psi_{i}}{\partial q^{\prime \prime}}(x) \frac{\partial}{\partial p^{\prime \prime}}, i=1, \ldots, r\right\} .
\end{aligned}
$$

ДокАЗАТЕльство. Касательное пространство к ребру $x_{1} \times \mathbb{R}^{m}$ принадлежит касательному пространству $\mathrm{k} l_{H}$, представимому в виде прямой суммы подпространства $\mathbb{R} \partial / \partial p_{0}$ (направления характеристики подмногообразия $\left.H^{(1)}\right)$ и касательного пространства $\mathrm{K} l_{\Gamma}$ (образа многообразия $l_{H}$ при проектировании вдоль характеристик подмногообразия $\left.H^{(1)}\right)$. Подмногообразие $l_{\Gamma}-$ лагранжево подмногообразие симплектического пространства, принадлежащее гиперповерхностям $\psi_{i}=0, i=1, \ldots, r$; следовательно, его касательные пространства состоят из характеристических направлений этих гиперповерхностей. Эти направления задаются гамильтоновыми векторными полями с гамильтонианами $\psi_{i}$. Предложение доказано.

Из условий трансверсальности (ребро $x_{1} \times \mathbb{R}^{m}$ трансверсально $\left\{p_{0}=p_{1}=0\right\}$ ) следует, что существует $\psi_{i}$ (скажем, $\left.\psi_{1}\right)$, такое, что вектор гамильтонова поля

$$
\frac{\partial \psi_{1}}{\partial p_{1}}(x) \frac{\partial}{\partial Q_{1}}+\frac{\partial \psi_{1}}{\partial p^{\prime \prime}}(x) \frac{\partial}{\partial q^{\prime \prime}}-\frac{\partial \psi_{1}}{\partial Q_{1}}(x) \frac{\partial}{\partial p_{1}}-\frac{\partial \psi_{1}}{\partial q^{\prime \prime}}(x) \frac{\partial}{\partial p^{\prime \prime}}
$$

трансверсален гиперповерхности $\left\{p_{1}=0\right\}$, т. е. $\partial \psi_{1} / \partial Q_{1}(x) \neq 0$. По теореме о неявной функции

$$
\begin{aligned}
l_{H}=\left\{\left(p_{0}, Q_{1}, p_{1}, q^{\prime \prime}, p^{\prime \prime}\right) \in H^{(1)} \mid Q_{1}=f_{1}\left(p_{1}, q^{\prime \prime}, p^{\prime \prime}\right)\right. \\
\left.\quad f_{i}\left(p_{1}, q^{\prime \prime}, p^{\prime \prime}\right)=\psi_{i}\left(f_{1}\left(p_{1}, q^{\prime \prime}, p^{\prime \prime}\right), p_{1}, q^{\prime \prime}, p^{\prime \prime}\right)=0, i=2, \ldots, r\right\} .
\end{aligned}
$$

В координатном $\left(p_{1}, q^{\prime \prime}, p^{\prime \prime}\right)$-пространстве $\mathbb{R}^{2 n-3}$ рассмотрим многообразие $l^{\prime}=\left\{\left(p_{1}, q^{\prime \prime}, p^{\prime \prime}\right) \mid f_{2}\left(p_{1}, q^{\prime \prime}, p^{\prime \prime}\right)=\cdots=f_{r}\left(p_{1}, q^{\prime \prime}, p^{\prime \prime}\right)=0\right\}$. Многообразие $l_{H}$ получается из $l^{\prime}$ вложением в гиперповерхность $\left\{Q_{1}=f_{1}\left(p_{1}, q^{\prime \prime}, p^{\prime \prime}\right)\right\}$ и умножением на $p_{0}$-ось, т.е. $l^{\prime} \cong l \times \mathbb{R}^{m-1}$. Из условий теоремы следует, что ребро $x_{1} \times \mathbb{R}^{m-1}$ трансверсально гиперповерхности $\left\{p_{1}=0\right\}$. Следовательно, $x_{1} \times \mathbb{R}^{m-1}$ трансверсально гиперповерхности $\left\{p_{1}=\varepsilon\right\}$ для любого малого $\varepsilon$, т. е. пересечения $l^{\prime} \cap\left\{p_{1}=0\right\}\left(\cong l \times \mathbb{R}^{m-2}\right)$ и $l^{\prime} \cap\left\{p_{1}=\varepsilon\right\}\left(\cong l \times \mathbb{R}^{m-2}\right)$ локально диффеоморфны: существует диффеоморфизм $g_{\varepsilon}:\left\{p_{1}=0\right\} \rightarrow\left\{p_{1}=\varepsilon\right\}$, переводящий $\left\{\left(q^{\prime \prime}, p^{\prime \prime}\right) \mid f_{i}\left(0, q^{\prime \prime}, p^{\prime \prime}\right)=0, i=2, \ldots, r\right\}$ в $\left\{\left(q^{\prime \prime}, p^{\prime \prime}\right) \mid f_{i}\left(\varepsilon, q^{\prime \prime}, p^{\prime \prime}\right)=0\right.$, $i=2, \ldots, r\}$. Диффеоморфизм

$$
g: H^{(1)} \rightarrow H^{(1)}, \quad g:\left(p_{0}, Q_{1}, p_{1}, q^{\prime \prime}, p^{\prime \prime}\right) \mapsto\left(p_{0}, Q_{1}, p_{1}, g_{p_{1}}^{-1}\left(q^{\prime \prime}, p^{\prime \prime}\right)\right),
$$

приводит многообразие $l_{H}$ к виду

$$
\begin{array}{r}
l_{H}=\left\{\left(p_{0}, Q_{1}, p_{1}, q^{\prime \prime}, p^{\prime \prime}\right) \in H^{(1)} \mid Q_{1}=f_{1}\left(p_{1}, g_{p_{1}}\left(q^{\prime \prime}, p^{\prime \prime}\right)\right)=\varphi\left(p_{1}, q^{\prime \prime}, p^{\prime \prime}\right),\right. \\
\left.f_{i}\left(0, q^{\prime \prime}, p^{\prime \prime}\right)=0, i=2, \ldots, r\right\} .
\end{array}
$$


Таким образом,

$$
\begin{aligned}
& l_{H}=\left\{(q, p) \in T^{*} M \mid q_{0}=0, q_{2}=-4 p_{0}^{3}-2 p_{1} p_{0}, p_{2}=3 p_{0}^{4}+p_{1} p_{0}^{2},\right. \\
& \left.q_{1}=-12 p_{0}^{5} / 5-2 p_{1} p_{0}^{3} / 3+\varphi\left(p_{1}, q^{\prime \prime}, p^{\prime \prime}\right), f_{i}\left(0, q^{\prime \prime}, p^{\prime \prime}\right)=0, i=2, \ldots, r\right\} .
\end{aligned}
$$

Заметим, что использовавшиеся выше преобразования не сохраняли симплектическую структуру, но сохраняли расслоение $\left(p_{0}, q_{1}, \ldots, q_{n}, p_{1}, \ldots, p_{n}\right) \mapsto$ $\left(q_{1}, \ldots, q_{n}, p_{1}, \ldots, p_{n}\right)$ на характеристики гиперповерхности $H$.

Проведя замену $Q=q_{1}-\varphi\left(p_{1}, q^{\prime \prime}, p^{\prime \prime}\right)$ и проектируя $l_{H}$ вдоль характеристик гиперповерхности $H$ (забывая $\left.p_{0}\right)$, получим

$$
\begin{gathered}
l_{M}=\left\{\left(Q, q_{2}, \ldots, q_{n}, p_{1}, \ldots, p_{n}\right) \mid f_{2}\left(0, q^{\prime \prime}, p^{\prime \prime}\right)=\cdots=f_{r}\left(0, q^{\prime \prime}, p^{\prime \prime}\right)=0,\right. \\
\text { многочлен } p_{0}^{5} / 5+p_{1} p_{0}^{3} / 3+q_{2} p_{0}^{2} / 2+p_{2} p_{0}+Q / 2 \text { от } p_{0} \\
\text { имеет корень кратности } \geqslant 3\} .
\end{gathered}
$$

Теорема доказана.

Автор благодарен В. М. Закалюкину за внимание к работе.

\section{ЛиТЕРАТУРА}

1. Арнольд В. И. Лагранжевы многообразия с особенностями, асимптотические лучи и раскрытый ласточкин хвост. Функц. анализ и его прил., 15, вып. 4, $1-14$ (1981).

2. Гивенталь А. Б. Особые лагранжевы многообразия и их лагранжевы отображения. В кн.: Соврем. пробл. матем. Новейшие достижения. Т. 33, 1988, c. $55-112$.

3. Щербак О. П. Волновые фронты и группы отражений. УМН, $\mathbf{4 3}$, вып. 3, 125160 (1988).

4. Мясниченко O. М. Геодезические границы в задаче об обходе препятствия и раскрытые ласточкины хвосты. Функц. анализ и его прил., 29, вып. 1, 82-84 (1995).

5. Melrose R. Equivalence of glancing hypersurfaces. Invent. Math., 37, 165-191 (1976).

Московский авиационный институт

Поступило в редакцию 7 апреля 1995 г. 